\title{
EPKM:An Efficient and Practical Key Management Scheme for Hierarchical Wireless Sensor Networks
}

\author{
Yang Wei, Qi Yue, Luo Xinqiang, Wang Qin \\ School of Computer and Communication Engineering \\ University of Science and Technology Beijing \\ Beijing, China \\ ustbyangwei@139.com
}

\begin{abstract}
Wirless sensor networks are becoming increasingly attactiv for military,wildlife monitoring and other applications. Usually the sensor nodes are deployed in a dangerous and untrusted area.Therefore,network security becomes very important.Key management is the core areas of the security. In this paper,we describe EPKM(Efficient and Practical Key Management Scheme), a key management protocol for the hierarchical wireless sensor networks. EPKM adopts the main idea of multiple keying mechanism which includes key establishment and updating procedures.It supports four types of keys for each node-an individual key shared with the gateway, a data encryption key shared with another node, a cluster key shared with the cluster head,and a global key that is initially preloaded by all the nodes. Compared to other key management schemes,our scheme used for establishing and updating these keys is efficient and practical as well as low storage and communication.
\end{abstract}

Keywords-wireless sensor networks; key management; hierarchical

\section{INTRODUCTION}

A Wireless Sensor Network (WSN for short) is composed of hundreds, even thousands of small sensor nodes, and can be widely used in many applications, for instance, military sensing, environment monitoring, medica treatment and industrial monitoring. WSN are usually deployed in a dangerous and untrusted area. Hence, security mechanism becomes very important for the operation of sensor applications [1]. Due to the characteristics of WSN, it has such limitations as limited battery power, finite computing and memory capability. Public key algorithms are typically considered to be too computationally intensive for resource constrained WSN nodes [2]. Thus, most of key management protocols for WSN are often based on symmetric key algorithms.

In this paper, we design a multiple keying mechanism for the hierarchical WSN based on symmetric key algorithms.It is suitable for meeting different security requirements. It supports four types of keys for each node-an individual key shared with the gateway, a data encryption key shared with another node, a cluster key shared with the cluster head,and a global key that is initially preloaded by all the nodes. The process of establishing and updating these keys is discussed in detail. Compared to other key management schemes,our scheme used for establishing and updating these keys is efficient and practical as well as low storage and communication.

The paper is organized as follows. Some existing key management approaches are reviewed in Section II. In Section III, details of EPKM approach are presented. In Section IV, the security and performance of EPKM are analyzed. Finally, it concludes the paper and outlines our future research plan.

\section{RELATED WORK}

In order to meet the need of practical applications,researchers have proposed a number of key management schemes for the hierarchical WSN. For example, Sencun et al.[3] proposed LEAP+(Localized Encryption and Authentication Protocol) scheme.It used different keys to secure different types of communication messages in the WSN.Also it supported Inter-node Traffic Authentication.It can lead to prevent the difficulty of launching many security attacks.But the entire network would suffer a severe loss if the aninitial key was exposed to an attacker.Jang et al.[4] proposed a Time-Based scheme.It proposed a secure scheme with a new notion of probabilistic time intervals.But its assumption of security in the initial deployment phase is not viable in many cases.Jia et al.[5] proposed a novel key management scheme.It adopted the main idea of threshold secret sharing scheme to divide the master key into several sub keys.It can provide strong security.But it increased computation complex.

Though these schemes have their own advantages, they can not apply to all situation in the hierarchical WSN.Our scheme provide different security requirements according to different message exchanged in WSN. It supports four types of keys for each node based on multiple keying mechanism. Furthermore, our scheme is very efficient and practical.

\section{Key Management Scheme}

Before introducing our protocol, we must describe some assumptions regarding the sensor network scenarios in which our protocols will be used:

(1) The sensor network is static and the topology is a hierarchical network .

(2) The gateway nodes is located in a well protected place and supplied with long-lasting power.It have sufficient memory resources .Also it is capable of detecting intrusion. 


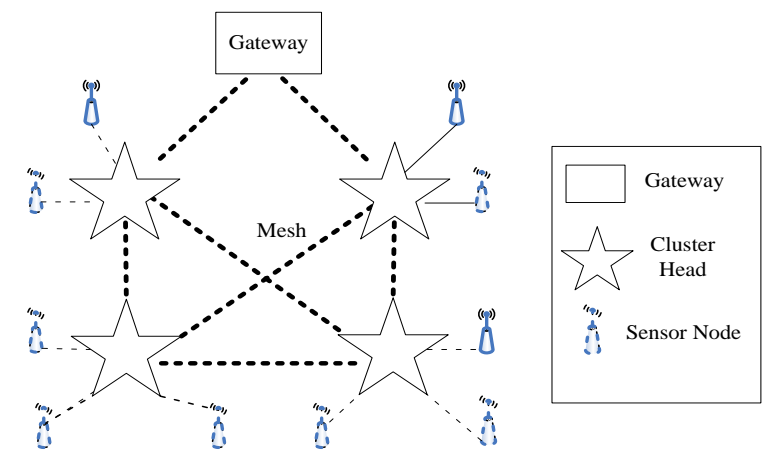

Figure 1. Hierarchical wireless sensor network model

\section{A. Overview}

We adopt the sensor network model proposed by Wireless Networks for Industrial Automation (WIA)[6].As illustrated in Fig.1, Our network supports a hierarchical network topology that is hybrid star and mesh. The first level of the network is in mesh topology where gateway nodes $(\mathrm{GW})$ and cluster head nodes $(\mathrm{CH})$ are deployed. The second level of the network is in star topology where cluster head nodes $(\mathrm{CH})$ and sensor nodes(SN) are deployed.

$\mathrm{GW}$ is a control center and used to connect the WSN with the other network. $\mathrm{CH}$ is responsible for constructing the star network and management of all the nodes in the cluster. Meanwhile, $\mathrm{CHs}$ can communicate with each other and forwarding data between its cluster members and the BS. $\mathrm{SN}$ can collect field data and send the data to its $\mathrm{CH}$. It only communicate with its $\mathrm{CH}$ directly.

The packets exchanged by nodes in WSN can be classified into several categories, e.g. beacons packets, control packets ,data packets and broadcast packets. Different type of packets requirements for Different security which depend on the category it falls in.

Global Key(KG) : Every node is initially preloaded with it before the network deployment. It is a globally shared key that is used by the GW and $\mathrm{CH}$ for encrypting securing broadcast messages.For example, A new node which wants to successfully join to the network have to listen to beacons from the $\mathrm{GW}$ or $\mathrm{CH}$ according to IEEE 802.15.4 standard[7].So only the legal node can decrypt the beacons and successfully join to the network.However, since the KG is shared among all the nodes, we have to design an effcient rekeying mechanism $i$ for updating it if a compromised node is revoked.

Individual Key (KI): Every node has a unique key that shares with the GW. The key is used for secure communication between a node and the GW. For example, a node may send an alert data to the GW if it observes any abnormal network conditions. Similarly, the GW can use the key to encrypt some sensitive information, e.g. special command or keying material.

Data Encryption Key(KED): In the EPKM, it supports a hierarchical network topology that is hybrid star and mesh. Every node which in mesh topology shares a KED with its immediate neighbors. However,the SN only shares a KED with its $\mathrm{CH}$. The keys are used to protect data and check integrity during the data transport. For example, a $\mathrm{CH}$ can use its KED to secure the distribution of its $\mathrm{KC}$ to its $\mathrm{SN}$, or to secure the transmissions messages to its neighbors $\mathrm{CH}$.

Cluster $\operatorname{Key}(\mathbf{K C})$ : In the EPKM,The second level of the network is in star topology where cluster head nodes $(\mathrm{CH})$ and sensor nodes( $\mathrm{SN})$ are deployed.A $\mathrm{KC}$ is a key shared by a $\mathrm{CH}$ and all its $\mathrm{SN}$, and it is mainly used for securing locally broadcast messages. Although we can use KED to encrypt the messages to every SN,it may cost to much energy consumption. Similarly,our purpose is for saving energy consumption in WSN.

Descriptions of the notations involved in this paper are listed as Table I.

TABLE I. NOTATION USED IN OUR SCHEME

\begin{tabular}{l|l}
\hline \multicolumn{1}{c|}{ Notion } & \multicolumn{1}{c}{ Description } \\
\hline $\mathrm{GW}$ & gateway node \\
\hline $\mathrm{CH}_{\mathrm{i}}$ & cluster head node $\mathrm{i}$ \\
\hline $\mathrm{S}_{\mathrm{i}}$ & the sensor node $\mathrm{i}$ \\
\hline $\mathrm{KG}$ & Global Key \\
\hline $\mathrm{KI}_{\mathrm{i}}$ & Individual Key of device $\mathrm{i}$ \\
\hline $\mathrm{K}_{\mathrm{ED}(\mathrm{i}, \mathrm{j})}$ & Data Encryption Key shared between device I and $\mathrm{j}$ \\
\hline $\mathrm{KC}$ & Cluster Key \\
\hline $\mathrm{MAC}(\mathrm{K}, \mathrm{M})$ & message authentication code for M using key $\mathrm{K}$ \\
\hline $\mathrm{N}$ & A nonrepeating value,such as an increasing counter \\
\hline $\mathrm{E}(\mathrm{K}, \mathrm{M})$ & the symmetric encryption for M using key $\mathrm{K}$ \\
\hline$\|$ & concatenation operator \\
\hline
\end{tabular}

\section{B. Key establishment}

Before network deployment, each node is initially preloaded with an initial $\mathrm{KG}$, 64-bit Unique Identifier address and $\mathrm{CBC}-\mathrm{MAC}(\mathrm{Cipher}$ Block Chaining-Message Authentication Code) function.

1) Establishing KI: Each node has a unique key that it shares with the GW. It is generated as follows: $\mathrm{KI}=\mathrm{MAC}(\mathrm{KG}, \mathrm{M})$.Here $\mathrm{M}$ is a node 64-bit Unique Identifier address combin with GW 64-bit Unique Identifier address. $\mathrm{KG}$ is pre-loaded into each node.So the node and $\mathrm{GW}$ can computer the same $\mathrm{KI}$ use the MAC function.Here MAC is the abbreviation of CBC-MAC function.

2) Establishing KED: Every node shares a KED with its immediate neighbors. However,the SN only shares a KED with its $\mathrm{CH}$.The keys are used to protect data during the data transport.

A new $\mathrm{CH}$ that wants to establish KED shall undergo the following steps.

a) The $\mathrm{GW}$ and Online $\mathrm{CH}$ periodicity broadcast beacon(BE) frame according to IEEE 802.15.4 standard.The new CHi keeps scanning the available channels until it has successfully received $\mathrm{BE}$ from $\mathrm{GW}$ or Online CH.It then tries to discover its one-hop neighbors. $\mathrm{GW} \rightarrow *: \mathrm{E}(\mathrm{KG}, \mathrm{BE} \| \mathrm{NA}) \| \mathrm{MAC}(\mathrm{KG}, \mathrm{E}(\mathrm{KG}, \mathrm{BE} \| \mathrm{NA}))$ 


\section{$\mathrm{CH} \rightarrow *$ : $\mathrm{E}(\mathrm{KG}, \mathrm{BE} \| \mathrm{NA}) \| \mathrm{MAC}(\mathrm{KG}, \mathrm{E}(\mathrm{KG}, \mathrm{BE} \| \mathrm{NA}))$}

b) For example, The new $\mathrm{CH}_{\mathrm{i}}$ discover two neighbors $\mathrm{CH}_{\mathrm{j}}$ and $\mathrm{CH}_{\mathrm{k}}$.It chooses an $\mathrm{CH}_{\mathrm{j}}$ as the temporal parent.It make use of the KI to encrypt the neighbors ID and $\mathrm{N}_{\mathrm{A}}$ then transmit to the $\mathrm{CH}_{\mathrm{j}}$. The $\mathrm{CH}_{\mathrm{j}}$ will forward a joining network request to the $\mathrm{GW}$.

$$
\begin{gathered}
\mathrm{CH}_{\mathrm{i}} \rightarrow \mathrm{CH}_{\mathrm{j}}: \mathrm{E}\left(\mathrm{KI}_{\mathrm{i}}, \mathrm{CH}_{\mathrm{i}}\left\|\mathrm{CH}_{\mathrm{j}}\right\| \mathrm{CH}_{\mathrm{k}} \| \mathrm{N}_{\mathrm{A}}\right) \| \operatorname{MAC}\left(\mathrm{KI}_{\mathrm{i}}, \mathrm{E}\left(\mathrm{KI}_{\mathrm{i}},\right.\right. \\
\left.\left.\mathrm{CH}_{\mathrm{i}}\left\|\mathrm{CH}_{\mathrm{j}}\right\| \mathrm{CH}_{\mathrm{k}} \| \mathrm{N}_{\mathrm{A}}\right)\right) \\
\mathrm{CH}_{\mathrm{j}} \rightarrow \ldots \rightarrow \mathrm{GW}: \mathrm{E}\left(\mathrm{KI}_{\mathrm{i}}, \mathrm{CH}_{\mathrm{i}}\left\|\mathrm{CH}_{\mathrm{j}}\right\| \mathrm{CH}_{\mathrm{k}} \| \mathrm{N}_{\mathrm{A}}\right) \| \mathrm{MAC}\left(\mathrm{KI}_{\mathrm{i}},\right. \\
\left.\mathrm{E}\left(\mathrm{K}_{\mathrm{jj}}, \mathrm{CH}_{\mathrm{i}}\left\|\mathrm{CH}_{\mathrm{j}}\right\| \mathrm{CH}_{\mathrm{k}} \| \mathrm{N}_{\mathrm{A}}\right)\right)
\end{gathered}
$$

c) The $\mathrm{GW}$ receives the joining request from the $\mathrm{CH}_{\mathrm{i}}$. Then it have to complete authentication process.if the $\mathrm{CH}_{\mathrm{i}}$ has been authenticated to be a legal device.The GW will allocation of network resources and distributthe KED to it.Also, the neighbors $\mathrm{CH}_{\mathrm{j}}$ and $\mathrm{CH}_{\mathrm{k}}$ will receive the $\mathrm{KED}$.

$\mathrm{GW} \rightarrow \mathrm{CH}_{\mathrm{i}}: \mathrm{E}\left(\mathrm{KI}_{\mathrm{i}}, \mathrm{K}_{\mathrm{ED}(\mathrm{CHi}, \mathrm{GW})}\|\ldots\| \mathrm{K}_{\mathrm{ED}(\mathrm{CHi}, \mathrm{CHj})}\|\ldots\|\right.$

$\left.\mathrm{K}_{\mathrm{ED}(\mathrm{CHi}, \mathrm{CHk})} \| \mathrm{N}_{\mathrm{A}}\right) \| \mathrm{MAC}\left(\mathrm{KI}_{\mathrm{i}}, \mathrm{E}\left(\mathrm{KI}_{\mathrm{i}}, \mathrm{K}_{\mathrm{ED}(\mathrm{CHi}, \mathrm{GW})}\|\ldots\|\right.\right.$ $\left.\mathrm{K}_{\mathrm{ED}(\mathrm{CH}, \mathrm{CH})}\|\ldots\| \mathrm{K}_{\mathrm{ED}(\mathrm{CHi}, \mathrm{CHk})} \| \mathrm{N}_{\mathrm{A}}\right)$ )

$\mathrm{GW} \rightarrow \mathrm{CH}_{\mathrm{j}}: \mathrm{E}\left(\mathrm{KI}_{\mathrm{j}}, \mathrm{K}_{\mathrm{ED}(\mathrm{CHi}, \mathrm{CHj})} \| \mathrm{N}_{\mathrm{A}}\right) \| \mathrm{MAC}\left(\mathrm{KI}_{\mathrm{j}}, \mathrm{E}\left(\mathrm{KI}_{\mathrm{j}}\right.\right.$, $\left.\mathrm{K}_{\mathrm{ED}(\mathrm{CHi}, \mathrm{CHj})} \| \mathrm{N}_{\mathrm{A}}\right)$ )

$\mathrm{GW} \rightarrow \mathrm{CH}_{\mathrm{k}}: \mathrm{E}\left(\mathrm{KI}_{\mathrm{k}}, \mathrm{K}_{\mathrm{ED}(\mathrm{CH}, \mathrm{CHk})} \| \mathrm{N}_{\mathrm{A}}\right) \| \mathrm{MAC}\left(\mathrm{KI}_{\mathrm{k}}, \mathrm{E}\left(\mathrm{KI}_{\mathrm{k}}\right.\right.$, $\left.\mathrm{K}_{\mathrm{ED}(\mathrm{CH}, \mathrm{CHk})} \| \mathrm{N}_{\mathrm{A}}\right)$ )

A new SN that wants to establish KED shall undergo the following steps.

a) The Online $\mathrm{CH}$ periodicity broadcast $\mathrm{BE}$ frame.The new $S_{a}$ keeps scanning the available channels until it has successfully received $\mathrm{BE}$ from Online $\mathrm{CH}$.For example, The new $\mathrm{S}_{\mathrm{a}}$ discover $\mathrm{CH}_{\mathrm{i}}$ as the $\mathrm{CH}$. The new $\mathrm{S}_{\mathrm{a}}$ make use of the $\mathrm{KI}$ to encrypt the joining request and send to $\mathrm{CH}_{\mathrm{i}}$.

$\mathrm{CH}_{\mathrm{i}} \rightarrow *: \mathrm{E}\left(\mathrm{KG}, \mathrm{BE} \| \mathrm{N}_{\mathrm{A}}\right) \| \operatorname{MAC}\left(\mathrm{KG}, \mathrm{E}\left(\mathrm{KG}, \mathrm{BE} \| \mathrm{N}_{\mathrm{A}}\right)\right)$

$\mathrm{S}_{\mathrm{a}} \rightarrow \mathrm{CH}_{\mathrm{i}}: \mathrm{E}\left(\mathrm{KI}_{\mathrm{a}}, \mathrm{S}_{\mathrm{a}} \| \mathrm{N}_{\mathrm{A}}\right) \| \operatorname{MAC}\left(\mathrm{KI}_{\mathrm{a}}, \mathrm{E}\left(\mathrm{KI}_{\mathrm{a}}, \mathrm{S}_{\mathrm{a}} \| \mathrm{N}_{\mathrm{A}}\right)\right)$

b) The Online $\mathrm{CH}_{\mathrm{i}}$ receive the joining network request.Then it will forward encrypted message to the $\mathrm{GW}$.

$$
\begin{gathered}
\mathrm{CH}_{\mathrm{i}} \rightarrow \mathrm{CH}_{\mathrm{j}} \rightarrow \ldots \rightarrow \mathrm{GW}: \mathrm{E}\left(\mathrm{KI}_{\mathrm{a}}, \mathrm{S}_{\mathrm{a}} \| \mathrm{N}_{\mathrm{A}}\right) \| \operatorname{MAC}\left(\mathrm{KI}_{\mathrm{a}}, \mathrm{E}\left(\mathrm{KI}_{\mathrm{a}}\right. \text {, }\right. \\
\left.\left.\mathrm{S}_{\mathrm{a}} \| \mathrm{N}_{\mathrm{A}}\right)\right)
\end{gathered}
$$

c) The GW receives the joining request from the $\mathrm{S}_{\mathrm{a}}$. Then it have to complete authentication process. If the $\mathrm{S}_{\mathrm{a}}$ has been authenticated to be a legal device.The GW will allocation of network resources and distribut the KED to it.Also,the $\mathrm{CH}_{\mathrm{j}}$ and $\mathrm{S}_{\mathrm{a}}$ will receive the KED.

$$
\begin{aligned}
& \mathrm{GW} \rightarrow \mathrm{CH}_{\mathrm{i}}: \mathrm{E}\left(\mathrm{KI}_{\mathrm{i}}, \mathrm{K}_{\mathrm{ED}(\mathrm{CHi}, \mathrm{Sa})} \| \mathrm{N}_{\mathrm{A}}\right) \| \operatorname{MAC}\left(\mathrm{KI}_{\mathrm{i}}, \mathrm{E}\left(\mathrm{KI}_{\mathrm{i}},\right.\right.\left.\left.\mathrm{K}_{\mathrm{ED}(\mathrm{CHi}, \mathrm{Sa})} \| \mathrm{N}_{\mathrm{A}}\right)\right) \\
& \mathrm{GW} \rightarrow \mathrm{S}_{\mathrm{a}}: \mathrm{E}\left(\mathrm{KI}_{\mathrm{a}}, \mathrm{K}_{\mathrm{ED}(\mathrm{CHi}, \mathrm{Sa})} \| \mathrm{N}_{\mathrm{A}}\right) \| \operatorname{MAC}\left(\mathrm{KI}_{\mathrm{a}}, \mathrm{E}\left(\mathrm{KI}_{\mathrm{a}},\right.\right. \\
&\left.\left.\mathrm{K}_{\mathrm{ED}(\mathrm{CHi}, \mathrm{Sa})} \| \mathrm{N}_{\mathrm{A}}\right)\right)
\end{aligned}
$$

3) Establishing $\mathrm{KC}: \mathrm{A} \mathrm{KC}$ is a key shared by a $\mathrm{CH}$ and all its $\mathrm{SN}$ in the same cluster.We use a very straightforward way to establish the key.The cluster head $\mathrm{CH}_{\mathrm{i}}$ wants to establish a $\mathrm{KC}$ with all its $\mathrm{SN}$.The $\mathrm{CH}_{\mathrm{i}}$ generates a random $\mathrm{KC}$, then encrypts the key with the KED between $\mathrm{CH}_{\mathrm{i}}$ and $\mathrm{SN}$ and then transmits the $\mathrm{KC}$ to each SN. Here, we take $\mathrm{CH}_{\mathrm{i}}$ and $\mathrm{S}_{\mathrm{a}}$ for example.

$$
\begin{gathered}
\mathrm{CH}_{\mathrm{i}} \rightarrow \mathrm{S}_{\mathrm{a}}: \mathrm{E}\left(\mathrm{K}_{\mathrm{ED}(\mathrm{CHi}, \mathrm{Sa})}, \mathrm{KC} \| \mathrm{N}_{\mathrm{A}}\right) \| \mathrm{MAC}\left(\mathrm{K}_{\mathrm{ED}(\mathrm{CHi}, \mathrm{Sa})}, \mathrm{E}\left(\mathrm{K}_{\mathrm{ED}}\right.\right. \\
(\mathrm{CHi}, \mathrm{Sa}) \\
\left.\left.\mathrm{KC} \| \mathrm{N}_{\mathrm{A}}\right)\right)
\end{gathered}
$$

Node $S_{a}$ receive the message and decrypts the key $\mathrm{KC}$. When one of the $\mathrm{SN}$ addition and deletion, the $\mathrm{CH}$ generates a new $\mathrm{KC}$ and transmits to all the remaining $\mathrm{SN}$ in the same way.

\section{Key updating}

Usually, the networks need periodic key updating mechanism to improve the security.Also, when a node is added or deleted ,it need to update the key.

1) Periodic Key updating: When the network exists for a certain period,it will start periodic key updating mechanism. The ISA100.11a which is a standard draft of industrial wireless measurement and control systems suggest that maximum lifetime of the keys is 48.5 days[8]. Surely,the lifetime of keys is configurable according to the application.In the EPKM,it suggest periodic update the KG and $\mathrm{KC}$.The periodic updating the $\mathrm{KG}$ is determined by the GW.The new KG is encrypted by the KI, and the encrypted new KG is distributed to the network device by the GW.The periodic updating the $\mathrm{KC}$ is determined by the $\mathrm{CH}$. The new $\mathrm{KC}$ is encrypted by the $\mathrm{KED}$, and the encrypted new $\mathrm{KC}$ is distributed to all the $\mathrm{SN}$ in the same cluster.

2) Node Addition: When a $\mathrm{SN}$ want to join the network,it fristly choose an Online $\mathrm{CH}$.The Online $\mathrm{CH}$ receive the joining network request. Then it will forward encrypted message to the GW.The GW will allocation of network resources and distribut the KED after it been authenticated to be a legal device.Also, The $\mathrm{KC}$ have to be updated in order to ensure backward confidentiality. The process of $\mathrm{KC}$ updating is the same as the establishing $\mathrm{KC}$.

3) Node Deletion: In the EPKM,it considers two case about node deletion.One is the SN deletion,the other is $\mathrm{CH}$ deletion. When one of SN deletion,the GW can detect the case as the $\mathrm{GW}$ periodically collects the $\mathrm{SN}$ health report.In order to ensure forward confidentiality, the GW sends key updating command to the $\mathrm{CH}$ which is the head of SN.The $\mathrm{CH}$ receive the command and trigger thekey update mechanism immediately. The process is the same as the establishing $\mathrm{KC}$.When one of $\mathrm{CH}$ deletion,the $\mathrm{GW}$ has a detection mechanism to know the case.If network have redundant $\mathrm{CH}$,it can quick use redundant $\mathrm{CH}$ after detecting the $\mathrm{CH}$ deletion.In order to ensure forward confidentiality, it only have to update the $\mathrm{KC}$. The process is the same as the establishing KC.However,most of WSN do not have redundant $\mathrm{CH}$. For example, the $\mathrm{S}_{\mathrm{a}}$ and $\mathrm{S}_{\mathrm{b}}$ have be distributed to $\mathrm{CH}_{\mathrm{i}}$ using the clustering algorithm. The process is illustrated as follows.

a) The GW send command to tell the other CH.The other $\mathrm{CH}$ delete the key relation to it and do not trust the message from it.

b) The GW distribute its $\mathrm{SN}$ among the remaining CH.It should be noted that the $\mathrm{CH}$ distribute the $\mathrm{SN}$ using some existing clustering algorithm. The GW distributes the new KED to the $\mathrm{CH}_{\mathrm{i}}, \mathrm{Sa}$ and $\mathrm{Sb}$.

$$
\begin{array}{r}
\mathrm{GW} \rightarrow \mathrm{CH}_{\mathrm{i}}: \mathrm{E}\left(\mathrm{KI}_{\mathrm{i}}, \mathrm{K}_{\mathrm{ED}(\mathrm{CHi}, \mathrm{Sa})}\left\|\mathrm{K}_{\mathrm{ED}(\mathrm{CHi}, \mathrm{Sa})}\right\| \mathrm{N}_{\mathrm{A}}\right) \| \operatorname{MAC}\left(\mathrm{KI}_{\mathrm{i}},\right. \\
\left.\mathrm{E}\left(\mathrm{KI}_{\mathrm{i}}, \mathrm{K}_{\mathrm{ED}(\mathrm{CHi}, \mathrm{Sa})}\left\|\mathrm{K}_{\mathrm{ED}(\mathrm{CHi}, \mathrm{Sa})}\right\| \mathrm{N}_{\mathrm{A}}\right)\right) \\
\mathrm{GW} \rightarrow \mathrm{S}_{\mathrm{a}}: \mathrm{E}\left(\mathrm{KI}_{\mathrm{a}}, \mathrm{K}_{\mathrm{ED}(\mathrm{CHi}, \mathrm{Sa})} \| \mathrm{N}_{\mathrm{A}}\right) \| \mathrm{MAC}\left(\mathrm{KI}_{\mathrm{a}}, \mathrm{E}\left(\mathrm{KI}_{\mathrm{a}},\right.\right. \\
\left.\left.\mathrm{K}_{\mathrm{ED}(\mathrm{CHi}, \mathrm{Sa})} \| \mathrm{N}_{\mathrm{A}}\right)\right)
\end{array}
$$




$$
\begin{gathered}
\mathrm{GW} \rightarrow \mathrm{S}_{\mathrm{b}}: \mathrm{E}\left(\mathrm{KI}_{\mathrm{b}}, \mathrm{K}_{\mathrm{ED}(\mathrm{CHi}, \mathrm{Sb})} \| \mathrm{N}_{\mathrm{A}}\right) \| \operatorname{MAC}\left(\mathrm{KI}_{\mathrm{b}}, \mathrm{E}\left(\mathrm{KI}_{\mathrm{b}}\right. \text {, }\right. \\
\left.\left.\mathrm{K}_{\mathrm{ED}(\mathrm{CH}, \mathrm{Sb})} \| \mathrm{N}_{\mathrm{A}}\right)\right)
\end{gathered}
$$

c) The $\mathrm{KC}$ have to be updated in order to ensure backward confidentiality beacuse of the $\mathrm{SN}$ addition.

$$
\begin{aligned}
& \mathrm{CH}_{\mathrm{i}} \rightarrow \mathrm{S}_{\mathrm{a}}: \mathrm{E}\left(\mathrm{K}_{\mathrm{ED}(\mathrm{CH} i \mathrm{Sa})}, \mathrm{KC} \| \mathrm{N}_{\mathrm{A}}\right) \| \mathrm{MAC}\left(\mathrm{K}_{\mathrm{ED}(\mathrm{CHi}, \mathrm{Sa})}, \mathrm{E}\left(\mathrm{K}_{\mathrm{ED}}\right.\right. \\
& \left.(\mathrm{CHi}, \mathrm{Sa}), \mathrm{KC} \| \mathrm{N}_{\mathrm{A}}\right) \text { ) } \\
& \mathrm{CH}_{\mathrm{i}} \rightarrow \mathrm{S}_{\mathrm{b}}: \mathrm{E}\left(\mathrm{K}_{\mathrm{ED}(\mathrm{CHi}, \mathrm{Sb})}, \mathrm{KC} \| \mathrm{N}_{\mathrm{A}}\right) \| \mathrm{MAC}\left(\mathrm{K}_{\mathrm{ED}(\mathrm{CHi}, \mathrm{Sb})}, \mathrm{E}\left(\mathrm{K}_{\mathrm{ED}}\right.\right. \\
& \text { (CHi,Sb), } \left.\mathrm{KC} \| \mathrm{N}_{\mathrm{A}}\right) \text { ) }
\end{aligned}
$$

\section{SECURITY AND PeRformance ANALysis}

This section analyzes security and performances of the keying mechanisms.It can provide sufficient security and achieve well performances.Also, the results of simulation show that our scheme have lower storage than LEAP+.

\section{A. Security analysis}

In this section, we analyze the security of the keying mechanisms in EPKM.Every node is initially preloaded with a KG. So only the legal node can use it to successfully join the network. It prevents some illegal node to access. Every node has a unique key KI that shares with the GW. It can ensure secure communication between a node and the GW. So,any SN's compromise won't affect the secure communication between the other SN and CH.The adversary can not attack the whole network even though they obtaining the key.The GW distributes the KED to nodes by using of the KI to encrypt.So the KED is Secure.The KC is randomly generated and is distributed by using of the KED. The adversary can not easy to get it.It ensure secure the locally broadcast messages.In the node addition, The $\mathrm{KC}$ have to be updated in order to ensure backward confidentiality. The key updating mechanism is discussed in detail toin order to forward confidentiality.Conclusively, our scheme can provide sufficient security and can achieve perfect resilience.

\section{B. Performances analysis}

1) Storage cost analysis: In the EPKM,a node needs to keep four types of keys.The $\mathrm{SN}$ only storage one $\mathrm{KG}$,one $\mathrm{KI}$,one $\mathrm{KED}$ and one $\mathrm{KC}$. The $\mathrm{CH}$ storage one $\mathrm{KG}$, one $\mathrm{KI}$, one $\mathrm{KC}$ and lots of KED. The number of KED is according the network size and the average number of node in a cluster.e.g. the network size is $\mathrm{N}$ and the average number of node in a cluster is $\mathrm{B}$, the average number of keys for a $\mathrm{CH}$ have to storage is $\mathrm{B}+\mathrm{N} / \mathrm{B}+2$. Compared to other key management schemes LEAP+, it have to storage $3 \mathrm{~B}+2$ keys. As shown in Fig.2,our scheme have lower storage than LEAP+ as the number of node in a cluster increasing.

2) Communication cost analysis: For updating the $\mathrm{KC}$, The $\mathrm{CH}$ have to send it to all of $\mathrm{SN}$ in the cluster.The average number of keys a $\mathrm{CH}$ transmitsis equal to $\mathrm{B}-1$ for a cluster of size B.For updating the KG,The GW have to send it to all of nodes in the network. So,the average number of keys the GW transmitsis equal to $\mathrm{N}$ for a network of size N.The $\mathrm{CH}$ have toforward the key message to the SN.The average number of keys a $\mathrm{CH}$ transmitsis also equal to $\mathrm{B}-1$ for a cluster of size B.The average cost is not very large as the $\mathrm{KG}$ is periodic updating.

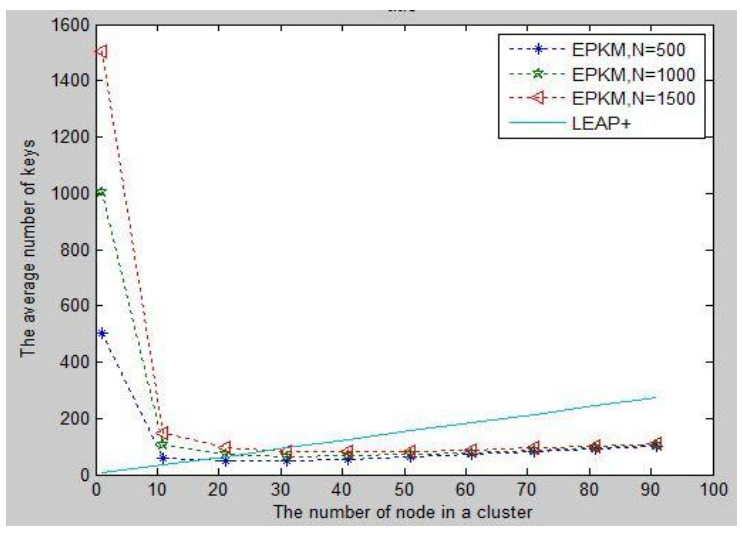

Figure 2. The average number of keys for $\mathrm{CH}$

\section{CONCLUSION}

In this paper,we propose EPKM scheme for hierarchical WSN.It adopts the main idea of multiple keying mechanism,design four types of keys for each node according to different message exchanged.The key establishment and key updating procedures used by EPKM are storage and communication effcient.Also it has strong security and perfect practicability.In our further research, we are going to improve its security to defend against various attacks.

\section{ACKNOWLEDGMENT}

The paper is sponsored by the National High Technology Research and Development Program of China (863 Program) (No. 2011AA0401), National Natural Science Foundation of China (No. 61003251).

\section{REFERENCES}

[1] Kavitha T, Sridharan D. Security vulnerabilities in wireless sensor networks: A survey[J]. Journal of information Assurance and Security, 2010, 5(1): 31-44.4

[2] Perrig A, Stankovic J, Wagner D. Security in wireless sensor networks[J]. Communications of the ACM, 2004, 47(6): 53-57.

[3] Zhu S, Setia S, Jajodia S. LEAP+: Efficient security mechanisms for large-scale distributed sensor networks[J]. ACM Transactions on Sensor Networks (TOSN), 2006, 2(4): 500-528.

[4] Jang J, Kwon T, Song J. A time-based key management protocol for wireless sensor networks[M].Information Security Practice and Experience. Springer Berlin Heidelberg, 2007: 314-328.

[5] $\mathrm{Hu}$ J, Bai E, Yang Y. A novel key management scheme for hierarchical wireless sensor networks[C].Communication Technology (ICCT), 2010 12th IEEE International Conference on. IEEE, 2010: 526-529.

[6] Liang W, Zhang X, Xiao Y, et al. Survey and experiments of WIA PA specification of industrial wireless network[J]. Wireless Communications and Mobile Computing, 2011, 11(8): 1197-1212.

[7] LAN/MAN Standards Committee. Part 15.4: wireless medium access control (MAC) and physical layer (PHY) specifications for low-rate wireless personal area networks (LR-WPANs)[J]. IEEE Computer Society, 2006.

[8] International Society of Automation. (2009). Wireless Systems for Industrial Automation: Process Control and Related Applications, ISA-100.11a-2009. [Online]. Available: http://www.isa.org 